\title{
RING NEBULAE AROUND Of AND WO STARS
}

\author{
Tatiana A. Lozinskaya \\ Sternberg State Astronomical Institute, Moscow, USSR
}

\section{Introduction}

To understand the origin of WR-rings it is of interest to consider nebulae associated with the precursors of WR stars and with stars at later stages of evolution. The former are represented by numerous Of stars and the latter by only four WO stars which, according to Barlow and Hummer (1982), are the most extreme WR stars near the end of their lives.

We can briefly summarize the properties and statistics of nebulae connected with Of stars, as compared with WR-rings, following Lozinskaya (1982; 1986):

i) About $80-90 \%$ of both WR and Of stars in a distance-complete sample are associated with $H I I$ regions, of which $30-40 \%$ have a ring-nebula-like morphology. By analogy to WR-rings, the Of-ring nebulae may be subdivided into three types: wind-blown bubbles, ejecta and shell-like $H I I$ regions; the characteristics of the WR and Of nebulae defining each type are similar.

ii) The three ways in which stars can influence the ISM - radiation, stellar winds and ejection of slow shells - work together to form multilayer shell-like structures of the ambient gas. The majority of the small-size bubbles and ejecta are located inside extended $H I I$ or $H I / C O$ shells or cavities. Many bubbles and ejecta which appear isolated are actually located inside supershells, like the WR ring nebula NCG 6888 inside the Cyg OB1 supershell.

What seems to be unclear regarding the origin of the shells is: why only $30-40 \%$ of stars with strong stellar winds are associated with ring nebulae contrary to theoretical predictions (the wind-blown bubbles are even more scarce, about $10 \%$ ).

One can see at least three explanations:

i)If a strong wind sweeps up, not the interstellar gas, but stellar ejecta of about $1-5 M_{\odot}$, the lifetime of a shell is determined by the emission measure of the expanding ejecta and may be shorter than the duration of the WR-stage. This explanation seems reasonable since the wind of the progenitor star and/or of a young stellar cluster may evacuate the ambient ISM.

ii) The short lifetime of wind-blown bubbles may be caused by a fast dissipation of the hot wind's energy due to hot plasma leakage through the shell, or cloud evaporation and so on.

iii) In a cloudy ISM the cold unshocked wind may leak through tenuous regions between dense clouds like fingers, without forming a shell-like structure. 


\section{Oxygen WR star environments}

The four Sanduleak WO stars are the most advanced massive stars, probably only several thousand years from a SN explosion. Indeed, our observations of WR 102 (Dopita et al., 1990; Dopita and Lozinskaya, 1990) indicate the absence of $\mathrm{He}$ in the star's envelope and point to the absence of it in the interior as well; the star's position on the HR diagram also supports our interpretation of it as the $C-O$ core of a massive star. Similarly, Sand 1 $\left[\mathrm{M}(\mathrm{WR})=14 M_{\odot}\right.$ and $\left.\mathrm{M}(\mathrm{O} 4)=50 M_{\odot}\right]$ seems to be the most evolved WR star known in a close binary (Moffat et al., 1985). Thus they provide a unique opportunity to investigate a SN-environment shortly before the explosion.

Since the WO stage is very short (the lifetime of the $C-O$ core of a massive star is only about $1 \%$ of the core- $\mathrm{He}$ burning duration) we cannot expect WO stars like WR 102 to constitute more than about $1 \%$ of the $\sim 300$ WR stars known; the four objects form quite a representative sample in the Galaxy and MCs.

WO stars are characterized by an extremely fast "superwind": $=v_{w}=\sim 4500$ to 7400 $\mathrm{km} \cdot \mathrm{s}^{-1}$ (Barlow and Hummer, 1982; Torres et al., 1986; Dopita et al., 1990) and the ambient gas is influenced by the previous "normal" outflow of the progenitor WR star. Both facts open new aspects for investigation into stellar wind vs. ISM interactions.

The four WO stars appear to be associated with optical and/or IR shell-like structures, although the short WO superwind does not participate in the shell's formation.

The nebula G2.4+1.4 around WR 102 is the only object of the four which was shown to be a classical wind-blown bubble (Dopita et al., 1990; Dopita and Lozinskaya, 1990 and these proceedings). We evaluate the star's mechanical luminosity as $L_{w}=(0.5-2) 10^{38} \mathrm{erg} \cdot \mathrm{s}^{-1}$ which is far in excess of the energy required to blow the shell $\left(R=5-6 p c, v_{\exp }=\right.$ $42 \mathrm{~km} \cdot \mathrm{s}^{-1}$, the ambient density ranges from $2-4$ to $30-60 \mathrm{~cm}^{-3}$ ). The shell's dynamical age $t \approx 10^{5} y r$ is much longer than the WO superwind duration, indicating that the progenitor star created the ring nebula before the onset of the WO stage. IRAS observations of the region reveal an incomplete ring coincident with the edge of the optical nebula, see Figure 1 and Graham (1985).

The second oxygen star in the Galaxy WR 142 is a member of the young open cluster Be 87. The cluster (distance $950 \pm 50 \mathrm{pc}$ ) is physically associated with an active starforming region ON2 (Turner and Forbes, 1982; Pitault, 1981) and both are located at the edge of a giant molecular cloud in front of Cyg X-Cyg OB1 (Cong, 1977). There is no prominent optical emission around WR 142 because of heavy absorption. Figure 2 shows the high resolution IRAS image of a small region around the star. It is confused by bright point sources (the two brightest are ON2 and Sh 106) and displays no signs of a shell-like morphology. The large-scale infrared images shown in Figure 3, however, reveal a prominent shell-like structure: a half-ring of about $3^{\circ}$ seen at both 60 and $100 \mu \mathrm{m}$. The reality of the IR shell is supported by a flat depression at $4.8 \mathrm{GHz}$ (see Wendker, 1984) and by a $C O$ arch-like feature (see Turner and Forbes, 1982; Dame et al., 1987).

The radius of the IR shell is about $23 \mathrm{pc}$; the measured dust colour temperature is $T(60 / 100)=29-30 K$; for a normal gas/dust ratio of 80 , we estimate its mass $M=$ $10^{3}(d / 1 k p c)^{2} M_{\odot}$ (Lozinskaya and Repin, 1990). Assuming it contains the gas evacuated from the interior, the initial density is $n_{o}=1 \mathrm{~cm}{ }^{-3}$. Using $L_{w}=(0.5-2) 10^{38} \mathrm{erg} \cdot \mathrm{s}^{-1}$ as a typical value for W01-WO2 stars, we evaluate the shell's age $t \approx(4.6-7.2) 10^{4} \mathrm{yr}$ using Dyson's model (Dyson, 1981), or longer if we take into account a small energy conversion efficiency. Here again the dynamical age seems to exceed the WO phase duration, leading to the conclusion that the short WO superwind does not participate in the shell's formation.

There is another WR star WR 143 inside this IR shell at a similar distance. Moreover, since the IR shell appears to confine the supershell around Cyg OB1 (Lozinskaya and Sitkin, 1988) we cannot exclude a physical connection. If this is the case, then there are 7-8 WR 

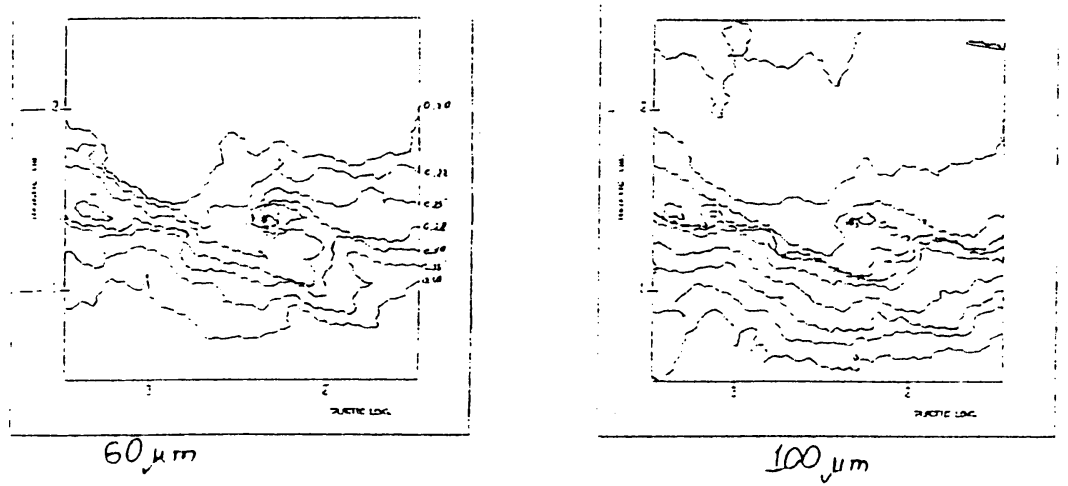

Fig.1. The IRAS 60 and 100 microns images of the region around WR 102

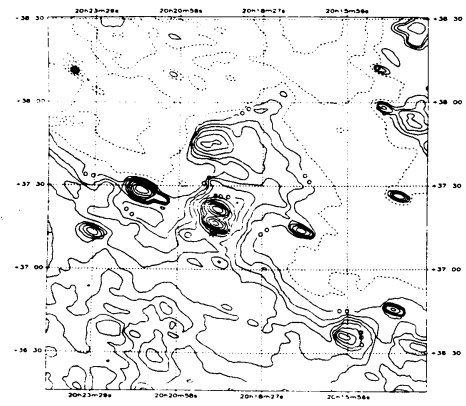

Fig. 2. The high resolution IRAS 60 microns image of a region around WR 142

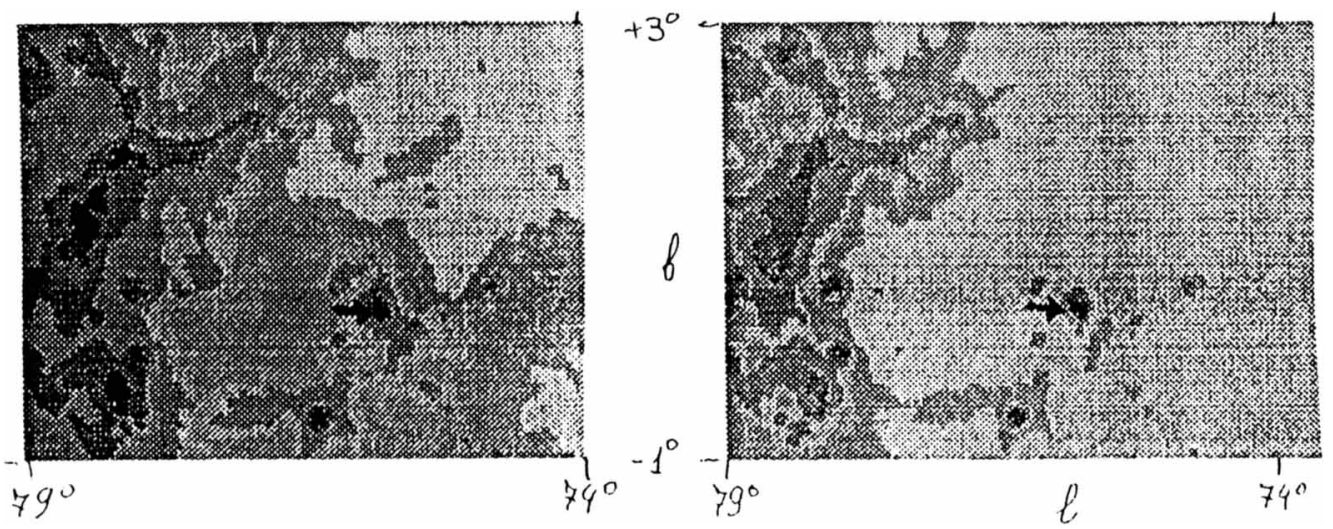

Fig. 3. Large-scale IRAS 60 microns image of a region around WR 142 
and Of stars spread over this large region, supplying ionizing and mechanical energy to the common shell. The mechanical luminosity of WR 142 is even sufficient to create the shell over the WR lifetime.

The environment of the WO4+02V binary Sand 1 in the SMC (the brightest star in NCG 602c) looks similar to WR 142. A weak thin filamentary shell DEM $167\left(35^{\prime}\right.$ in diameter) is easily seen on the photographs of the SMC around NCG 602c, while DEM 166 (N90), associated with NGC 602a,b, is a bright compact $H I I$ region on its southern edge (Westerlund, 1964; Davies et al., 1976). The high contrast masked print made by Meaburn (1980) demonstrates the complicated structure of his supershell SMC 1 with a smaller filamentary ring (about $30-40^{\prime}$ ) comprising NCG 602c and Sand 1. The IRAS imaging shows an evident ring of IR emission coinciding with the optical shell (see the IRAS $100 \mu m$ image of the SMC in Loiseau et al. (1987)).

Surely, Sand 1 is not the only blue star inside the supershell. However, the difference between the two HII regions - the compact one connected with NGC 602a,b, and the thin ring around NGC602c - may be considered as an indication of the influence of Sand 1. Indeed, NGC 602a,b and NGC 602c form a single association; their stellar contents (except Sand 1) and the associated gas masses: $M(D E M 166)=210^{4} M_{\odot}, M($ shell $)=310^{4} M_{\odot}$ are similar (Westerlund, 1964; Kontizas and Morgan, 1988). The stellar wind of Sand 1 is also powerful enough to create the shell. The $H I$ surface density in the region is of order $N(H)=1.210^{21}$ at $/ \mathrm{cm}^{-2}$ (McGee and Newton, 1982); the gas is uniformly spread in velocity (Mathewson et al., 1988), implying a uniform distribution along the line of sight. If the depth of the SMC wing is of order 5-10 kpc, the ambient density would be $\sim 0.04-0.08 \mathrm{~cm}^{-3}$. For the WR+04 wind energy output of $L_{w} \simeq 210^{38} \mathrm{erg} \cdot \mathrm{s}^{-1}$ $\left(<\dot{M}>(W R) \simeq 410^{-5} M_{\odot} y r^{-1}\right.$ (Lozinskaya and Sitkin, 1988); $v_{w}=4200 \mathrm{~km} \cdot \mathrm{s}^{-1}$ (Barlow and Hummer, 1982), the wind duration required to create a bubble of radius $260-300 p c$ is $t=(2.5-5) 10^{6} y r$, comfortably short for the massive system lifetime.

Sand 2 in the LMC appears to be superimposed on the faint diffuse filamentary $H I I$ region DEM 268 of size $65^{\prime} 40^{\prime}$ (Davies et al., 1976). A smaller filamentary ring comprising the WO star is easily seen of about $20^{\prime}(\sim 270 p c)$ extent. Several OB stars and SGs exist within the large shell DEM 268; excepting Sand 2 (Isserstedt, 1984), their combined UV emission rate and stellar winds are required to produce the shell-like nebula. Deeper photographs and kinematics are required to reveal the nature of the filamentary shell around Sand 2 more fully. The region is quite complicated; the very faint filaments near the 30 Doradus nebula between LMC3 and LMC4 outline a supershell as large as $\sim 1 \mathrm{deg}$ (Meaburn, 1980).

Despite the small number of WO stars and the very preliminary character of our examination we may conclude: The four WO stars are located inside optical and/or IR shell-like structures. Only the nebula G2.4+1.4 around WR102 is a classical wind-blown bubble but even here the short "superwind" of the oxygen sequence stage does not seem to be important in the shell's creation. We suppose that the progenitor star forms the ring nebula before the onset of the WO stage and the subsequent superwind produces the characteristic thin-filamentary "scalloped" structure as a result of impacting on the shell, probably accompanied by leakage of the superwind through the holes punched in the shell.

As for the stars inside the supershells, their evolutionary connections are not completely clear. On the one hand the winds of WR stars are sufficient to create the corresponding shells. In the tenous interior of a supershell around a young cluster, the short WO superwind acts as a supplementary energy source re-heating the superbubble.

On the other hand, perhaps a supershell's propagation into a dense molecular cloud will trigger supermassive star formation, a prerequisite to reaching the final oxygen-sequence stage in WR evolution. 


\section{References}

Barlow, M.J., Hummer, D.G., 1982, in IAU Symp.No.99. Wolf-Rayet Stars: Observations, Physics, Evolution. Eds. C.W.H. de Loore, A.J. Willis (Dordrecht: Reidel), p. 387.

Cong, H.I.L., 1977, Ph.D. Thesis, Columbia University.

Dame, T.M., Ungerechts, H., Cohen, R.S., de Geus, E.J., Gremier, I.A., May, J., Murphy, D.C., Nyman, L.-R., Thaddeus, P., 1987, Astrophys. J. 322, 706.

Davies, R.D., Elliott, K.H., Meaburn, J., 1976, Mem. R. astr. Soc. 81, 89.

Dopita, M.A., Lozinskaya, T.A., McGregor, P.J., Rawlings, S.J., 1990, Astrophys. J. 351, 563.

Dopita, M.A., Lozinskaya, T.A., 1990, Astrophys. J. 359, 419.

Dyson, J.E., 1981, in: Investigating the Universe. Ed. F.D. Kahn (Dordrecht: Reidel), p. 125.

Graham, J.R., 1985, Observatory 105, 7.

Isserstedt, J., 1984, Astron. Astrophys. 131, 347.

Kontizas, E., Morgan, D.H., 1988, Astron. Astrophys. 201, 208.

Loiseau, N., Klein, U., Greibe, A., Wielebinske, R., Haynes, R.F., 1987, Astron. Astrophys. 178, 62.

Lozinskaya, T.A., 1982, Astroph. Space Sci. 87, 313.

Lozinskaya, T.A., 1986, Supernovae and stellar winds: the interaction with the ISM, Moscow, Nauka (AIP, New York, 1991).

Lozinskaya, T.A., Repin, S.V., 1990, Astron. Zh. (in press).

Lozinskaya, T.A., Sitnik, T.G., 1988, Pis'ma Astron. Zh. 14, 240 (= Sov. Astron. Letters $14,100)$.

Mathewson, D.S., Ford, V.L., Visvanathan, N., 1988, Astrophys. J. 333, 617.

McGee, Newton, 1982, Proc. Astron. Soc. Austral. 4, 195.

Meaburn, J., 1980, Monthly Notices Roy. Astron. Soc. 192, 365.

Moffat, A.F.J., Breysacher, J., Seggewiss, W., 1985, Astrophys. J. $292,511$.

Pitault, A., 1981, Astron. Astrophys. 97, L5.

Torres, A.V., Conti, P.S., Massey, P., 1986, Astrophys. J. 300, 379.

Turner, D.G., Forbes, D., 1982, Publ. Astron. Soc. Pacific 94, 789.

Wendker, H.J., 1984, Astron. Astrophys. Suppl. 59, 291.

Westerlund, B.E., 1964, Monthly Notices Roy. Astron. Soc. 127, 429.

\section{DISCUSSION}

Cassinelli: Thank you very much. I have always liked that supermassive star idea... I noticed that some of your nebulae were very massive. From IRAS data you were deriving masses in the order of a $1000 M_{\odot}$.

Lozinskaya: The masses of all infrared shells look like something several times $10^{3} M_{\odot}$. You see, Dopita will provide the exact data, but today I am dealing with a large deal of speculation. I think that such a massive star may create a supershell. But on the other hand maybe it is the opposite: maybe a supershell is responsible for the creation of a supermassive star which becomes a WO star. 


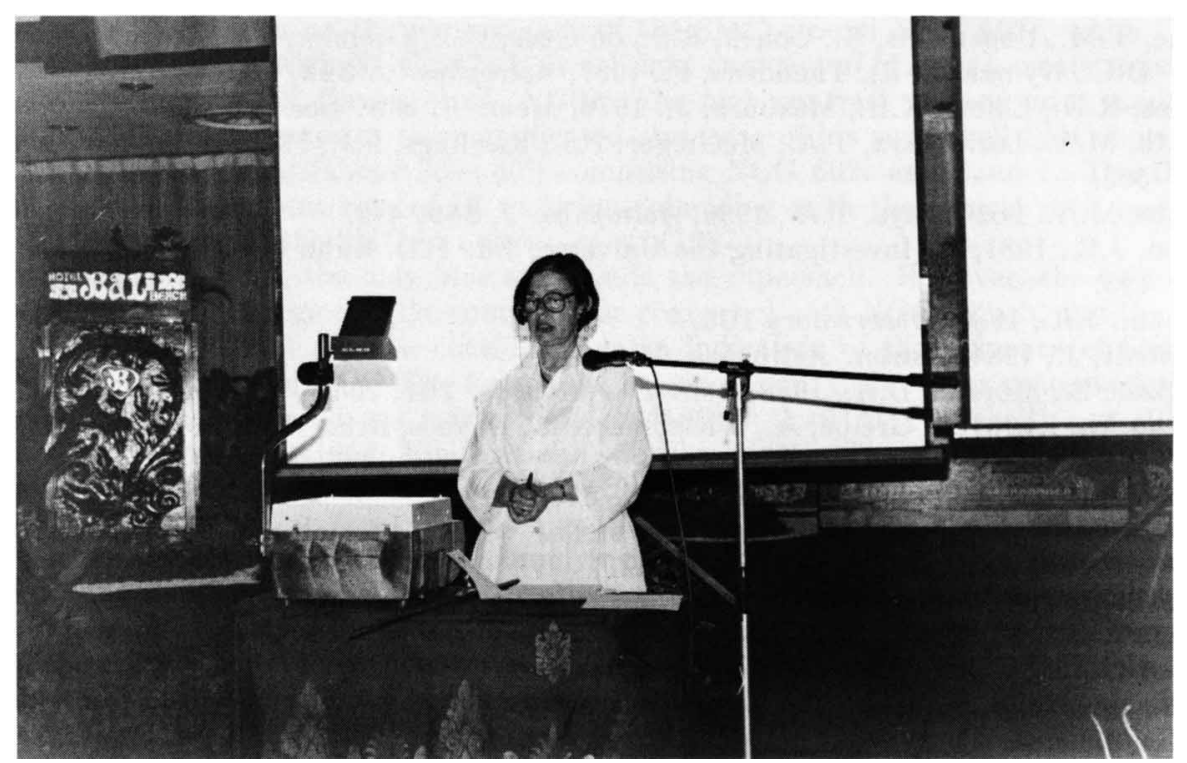

Tatiana Lozinskaya 